\title{
In situ Observation of Bulk 3D Grain Evolution During Plastic Deformation in Polycrystalline $\mathrm{Cu}$
}

\author{
Reeju Pokharel ${ }^{\mathrm{a}, \mathrm{c}, *}$, Jonathan Lind $^{\mathrm{a}, \mathrm{b}}$, Shiu Fai Li ${ }^{\mathrm{b}}$, Peter Keneseid ${ }^{\mathrm{d}}$, Ricardo A. Lebensohn ${ }^{\mathrm{c}}$, Robert M. Suter ${ }^{\mathrm{a}}$, \\ Anthony D. Rollett ${ }^{\mathrm{a}}$ \\ ${ }^{a}$ Carnegie Mellon University, 5000 Forbes Avenue, Pittsburgh PA 15213, USA \\ ${ }^{b}$ Lawrence Livermore National Laboratory, 7000 East Avenue, CA 94550 USA \\ ${ }^{c}$ Materials Science and Technology Division, Los Alamos National Laboratory, Los Alamos, NM 87544, USA \\ ${ }^{d}$ Advanced Photon Source, Argonne National Laboratory, 9700 South Cass Avenue, Argonne, IL 60439, USA
}

\begin{abstract}
We present a non-destructive in situ measurement of three-dimensional (3D) microstructure evolution of $99.995 \%$ pure polycrystalline copper during tensile loading using synchrotron radiation. Spatially resolved three-dimensional crystallographic orientation fields are reconstructed from the measured diffraction data obtained from a near-field high-energy X-ray diffraction microscopy (nf-HEDM), and the evolution of about 5000 3D bulk grains is tracked through multiple stages of deformation. Spatially resolved observation of macroscopic texture change, anisotropic deformation development, and the correspondence of different crystallographic parameters to defect accumulation are illustrated. Moreover, correlations between different crystallographic parameters, such as crystal rotation evolution, short- and long-range orientation gradient development, microstructural features, and grain size effects are investigated. The current state of data mining tools available to analyze large and complicated diffraction data is presented and challenges associated with extracting meaningful information from these datasets are discussed.
\end{abstract}

Keywords: High-energy X-ray diffraction microscopy, Non-destructive microstructure measurement, 3D microstructure response, Bulk grain evolution, Polycrystalline copper, Plastic deformation and anisotropy, Micromechanical modeling, Crystal plasticity

\section{Introduction}

The mechanical behavior of polycrystalline materials is complex in general, due to neighborhood-specific interactions between anisotropic crystalline grains. For each crystallite, its neighborhood's microstructure determines the local elastic and plastic responses to thermomechanical loads applied at aggregate level. While computational models that combine physics-based and heuristic rules are currently able to reproduce the evolution of averaged quantities such as stress-strain response and crystallographic texture, they are not able to fully reproduce the details of the various complex responses that are observed locally (Zhao et al., 2008; Shade et al., 2011). A recent review (Pokharel et al., 2014) surveys this limited agreement. In order to gain a more fundamental understanding, measurements are required to track, inside of bulk samples, the relevant quantities at the relevant length scales as loading is applied. It is only with such data, along with input from the nanoscale on the properties and responses of defect ensembles in individual grains that the development of truly predictive models will be accomplished (Zeghadi et al., 2007).

For example, the study of polycrystal deformation based on Taylor or self-consistent models is limited to considering homogeneous strain and lattice rotation of the grains in an aggregate. The development of orientational broadening in X-ray diffraction (Joffe and Kirpitcheva, 1922) and the obvious spread in measured orientation maps, however, makes it clear that each grain and each portion of a grain is affected by its neighbors (Pokharel et al., 2014; Li et al., 2012; Pouillier et al., 2012).

Dual and tri-beam microscopes have been developed that combine electron backscatter diffraction (EBSD) orientation mapping at surfaces (Schwartz et al., 2009) with automated serial sectioning by mechanical means (Rowenhorst and Lewis, 2011), ion beams (Uchic et al., 2011) and/or laser ablation (McDonald et al., 2008). These systems measure a sequence of layers that can then be assembled into three dimensional data sets, albeit

${ }^{*}$ Corresponding author.

Email address: reeju@lanl.gov (Reeju Pokharel) 
with significant effort to align the sections in order to compensate for drift and other limitations of this experimental technique. Thanks to the point-by-point electron beam probe with a spatial resolution as small as $0.1 \mu \mathrm{m}$, it is possible to characterize local lattice orientations in a wide variety of deformation states (Schwartz et al., 2009; Quey et al., 2012; Cerreta et al., 2013). However, the destructive nature of sectioning means that only single states of a given sample can be studied and deformation must be studied after the fact. Data collection is laborious and time-consuming and this limits the number of data sets that have been obtained for systematic studies. To capture the behavior of grains inside a bulk sample but still only characterizing a surface, experiments have been conducted on split samples; the results were similar, however, to those obtained on free surfaces or quasi-2D microstructures (Quey et al., 2012).

As an alternative to surface measurements, a variety of non-destructive synchrotron based X-ray diffraction techniques have been developed which provide both statistical and spatially resolved characterizations. White microbeam methods (Larson et al., 2002; Barabash et al., 2009) have submicron spatial resolution and, similar to EBSD, they provide point-by-point measurements even in highly disordered material states. 3D resolution can be obtained to depths of the order of tens of microns in many materials with sensitivity to local crystal orientations and strain states. High energy ( $\geq 40 \mathrm{keV}$ ) monochromatic X-ray methods have been developed with the aim of probing deep ( $1 \mathrm{~mm}$ ) inside of bulk materials (Poulsen et al., 2004; Lienert et al., 2011; Ludwig et al., 2008). Implementations referred to as 3DXRD (primarily at the European Synchrotron Radiation Facility) or HEDM (at the Advanced Photon Source) continue to be developed and new facilities are being developed at the PetraIII and the Cornell High Energy Synchrotron Source. Different experimental configurations (Poulsen et al., 2004; Poulsen, 2012) lead to sensitivity to lattice strains in individual grains (Bernier et al., 2011), or ensembles of grains (Bernier and Miller, 2006), or to spatially resolved orientations similar to EBSD but with 3D spatial resolution. These measurements can be combined with each other (Shade et al., 2013), with a variety of sample environments (Pokharel et al., 2014; Li et al., 2012; Shade et al., 2013; Lind et al., 2014; Hefferan et al., 2012), and with additional X-ray or optical probes (Li et al., 2012; Shade et al., 2013).

Here, we present a fully three-dimensional measurement and analysis of a polycrystalline microstructure as it undergoes ductile deformation under tensile loading. We use near-field HEDM (nf-HEDM) (Suter et al., 2006) to map lattice orientations in and between roughly 7000 grains in a $\sim 0.3 \mathrm{~mm}^{3}$ volume of a copper polycrystal as the sample is pulled in tension up to $21 \%$ true strain. The data provide local neighborhood information for each grain, both in the initial undeformed state and as deformation proceeds. We demonstrate the feasibility of extracting both global and local statistical information as well as the tracking of local regions between states. The data, only a portion of which are presented here (due to limited computational resources for data reduction/reconstruction), are amenable to direct comparisons to plasticity models and such comparisons are currently underway.

\section{Methods}

\subsection{Material $\mathcal{F}$ Experimental Set-up}

The $99.995 \%$ pure copper sample and in-situ experimental geometry used here are similar to those of (Pokharel et al., 2014). In that measurement, we sampled a single layer in 12 relatively finely spaced strain increments up to $14 \%$ macroscopic strain whereas here, we perform volumetric measurements at each of five strain levels up to $21 \%$ macroscopic strain. Fig. 1 shows a schematic of the sample and the tension apparatus (Pokharel et al., 2014; Lind et al., 2014) mounted on translation and rotation stages of the experimental facility at the 1-ID APS beamline. The stepping motor driven tension apparatus pulled the sample in steps of $5 \mu \mathrm{m}$ corresponding to strain steps of $\delta \epsilon \approx 5 \times 10^{-3}$. Fig. 2 shows the resultant macroscopic stress-strain curve.

\section{2. nf-HEDM Data Acquisition}

Table 1 shows characteristics of the five volumetric measurements that were collected in a single synchrotron run of three days. In all cases, the line focused, $\leq 4 \mu \mathrm{m} \times 1.3 \mathrm{~mm}$ X-ray beam illuminates a thin cross-section of the sample. After each "layer measurement" the sample is translated $4 \mu \mathrm{m}$ vertically to illuminate the next such layer, this process is repeated at any given fixed strain state until the full desired 3D volume has been scanned. Following a full volumetric scan, the tensile strain is increased, and additional layer measurements are added so as to span, as best as possible, the entire material volume measured in the initial, S0, state, hence the total volume measured increases with strain. However, the S4 measurement was truncated by the end of the beam time allocation and only part of the original volume was measured. In each volume, the measured layers were roughly centered at the middle of the $1 \mathrm{~mm}$ diameter gauge section of the sample. As in Pokharel et al. (2014), the center of the volumes was shifted to account for the displacement of the upper end of the sample by the tensile deformation apparatus. 
Recent advances in data acquisition are essential in making these large volume measurements feasible. Image data as described in Fig. 1 are now collected "on-the-fly" as the air bearing rotation stage rotates continuously. The interline CCD camera (Retiga 4000DC, which uses a Kodak KAI-4022 CCD with $2 \mathrm{k} \times 2 \mathrm{k}$ with $7.4 \mu \mathrm{m}$ pixels), is optically coupled through $5 \times$ magnifying optics so as to image fluorescent light from a $18 \mu \mathrm{m}$ thick, single crystal, Lutecium-Aluminum-garnet scintillator. On completion of an exposure, charge is rapidly transferred to readout wells associated with each pixel on the CCD after which collection of the next image begins and the previous one is read out. At typical rotation speeds $\left(\sim 0.3-1^{\circ} /\right.$ second $)$, only a negligible rotation interval $\left(\leq 0.001^{\circ}\right)$ is missed. Since the missed interval is less than the Darwin width of a typical perfect crystal Bragg peak, (Warren, 1969) essentially no diffraction signal is lost. Previously, a start and stop sequence that cost 1.7 seconds was associated with each image; with $\approx 36,000$ images per volume measurement, the new procedure saves 17 hours per volume relative to past work (Pokharel et al., 2014; Li et al., 2012; Lind et al., 2014; Hefferan et al., 2012). The entire raw data set comprises roughly 1.6 terabytes of image data. This was one of the first data sets collected with this new protocol and, not surprisingly, some interfacing issues were encountered. In particular, the first, S0, data set was mildly compromised as will be discussed in Sec. 2.3. However, as shown below, the data track $\sim 5,000$ bulk grains through four strain states, with a smaller number in the final, S4, data set. This is the largest data set collected to date with nf-HEDM at the APS that tracks the evolution of microstructure under plastic deformation (Pokharel et al., 2014; Li et al., 2012; Lind et al., 2014; Hefferan et al., 2012; Oddershede et al., 2011).

\subsection{Forward Modeling Method (FMM) Reconstructions of Microstructure}

Reconstruction of crystal orientations from the diffraction images is performed using the forward modeling method (FMM) (Suter et al., 2006; Li and Suter, 2013). Orientation maps presented in this paper have in plane resolution of $2.8 \mu \mathrm{m}$ and out of the plane resolution (perpendicular to the layers) is simply the layer spacing of 4 $\mu \mathrm{m}$. Examples of layer reconstructions in the different strain states are given in Fig. 2. The confidence parameter, $C$, is defined as the fraction of simulated Bragg peaks that overlap observed diffracted beams. In this work, the simulation included Bragg scattering up to $Q_{\max }=2 k \sin \theta_{\max }=8 \AA^{-1}$, with $k=\frac{2 \pi}{\lambda}=33.11 \AA^{-1}$, which includes scattering up to the $\{h l k\}=\{420\}$ family of peaks for copper with lattice constant, $a=3.61 \AA$. Over the $180^{\circ}$ experimental rotation range, a typical orientation generates $\approx 50$ peaks, with $Q \leq Q_{\max }$, that will strike the detector. Note also that the S0 state maps in Fig. 2 contain scattered black lines indicating local orientation fluctuations greater than $5^{\circ}$. Part of this is due, however, to the above mentioned data collection/interfacing problem: roughly $0.1 \mathrm{deg}$ of each $1 \mathrm{deg}$ integration interval is missing in each image frame. This resulted in increased orientation noise in the S0 state, which was cleaned up in the post processing step. As shown in Li and Suter (2013), the fidelity of forward model based orientation reconstructions degrades gracefully with increasing orientation variation within grains. All voxels considered here have orientations determined by overlap of simulated Bragg peaks with 20 or more experimentally observed diffracted beams (that is, $C \geq 0.4$ ). Thus, we expect presented trends to be robust. More quantitative error analysis and validation against deformed samples and simulated data sets is a substantial undertaking that is currently being pursued. Below, we make comparisons of trends to prior observations where they exist.

\subsection{Verification of $3 D$ Grain Tracking}

Before presenting detailed analysis in Sec. 3, we verify the extent to which the initial material volume measured in S0 is tracked through states S2 and S3 and that the structures are sufficiently similar that individual grains can be followed. Fig. 3 shows the three entire reconstructed volumes. These images correspond to the same vertical section extracted from a stack of spatially resolved, reconstructed orientations; no smoothing or alignment procedures have been applied. Reasonably smooth boundaries are observed both within the reconstruction planes (top surface; referred to as the $x y$-plane) and across the layers in the z-direction, i.e., spanning multiple independently measured and reconstructed layers. Fig. 4 shows cross-sectional cuts through the centers of the volumes in the $y z$ plane. Reduction in the cross-section diameter (horizontal extent of the figures) with increasing strain is evident. While grain cross-section shapes are generally quite similar, clear differences are evident. Such differences can be due to physical boundary motions due to tensile strain, the impossibility of picking "the same" planar section in states with different sample shapes, the voxelized nature of the reconstructions, and/or increasing errors in spatially resolving grains as deformation increases and signal quality decreases.

While uniformly colored regions in Figs. 3 and 4 are easily identified by eye as "grains," no such distinction has been made in the measurement or reconstruction. We are free to associate sets of voxels with similar orientation as belonging to such entities. Computationally, we associate connected voxels with small misorientations that are enclosed by orientation discontinuities larger than a threshold angle. Throughout the analysis presented here, we use a $5^{\circ}$ threshold. Fig. 4(d) shows the boundaries so deduced in the overlapping cross-sections of (a) through (c). 
Apparent boundary motions are quite variable but it is clear that many grain correspondences between states can be made.

To track grains in 3D at multiple strain levels, the grains in each state are initially sorted by volume, and only those that contain more than 100 voxels are tracked. For each grain in the initial state, all the grains in the deformed state are examined and the misorientation between average orientations and distance between center of mass $(\mathrm{COM})$ positions are computed in three dimensions. The grain that gives the minimum misorientation angle and the smallest COM distance is considered to be the same grain. To accept a correspondence, the misorientation must be $\leq 5^{\circ}$ and the COM distance must be $\leq 50$ voxels. Both threshold misorientation and COM have to be satisfied for it to be considered the same grain. One could also check for grain volumes but, owing to grain fragmentation during deformation, the number of grains that can be registered decreases with strain. Therefore, out of $\sim 7000$ grains, 4930 were registered between states and rest were not included in tracked grain analyses. Fig. 5 shows a 3D projection of a tracked grain across the three strain states. While there is substantial lattice rotation (not discernible for moderate strains with this coarse color scale), the grain shape is largely the same in each state. This confirms that the tracking algorithm reliably maps grains between initial and deformed stages.

\section{Results}

\subsection{Macroscopic Texture Evolution}

As is well known, plastic deformation applied to a polycrystal along a constant strain path changes the texture. Fig. 6 shows inverse pole figures computed using all measured voxels in each of states S0, S2, and S3. The orientations in the initial microstructure correspond to a nearly random texture with some weak peaks along $\langle 111\rangle$ and $\langle 001\rangle$ directions. As is known experimentally and can be understood by a simple Taylor analysis, lattice rotations strengthen the $\{111\}$ fiber component and, to a lesser extent the $\{100\}$ component, while decreasing the frequency of orientations near $\{110\}$ and $\{112\}$. While these trends are clear in the figures, the sample would best be called weakly textured even after $12 \%$ tensile strain.

\subsection{Anisotropic Plastic Deformation}

By contrast to the well understood nature of grain orientation evolution shown in Fig. 6, the more challenging goal of the the present study is to understand defect accumulation at a local level utilizing the full 3D measured volumes. It is obvious that macroscopic behavior is a manifestation of the collective behavior of ensembles of individual grains (Kalidindi et al., 2004) but many questions remain about how grains interact. Thus, making sense of the plastic response at the local, or grain and subgrain scales is crucial for understanding emergent behavior at the macroscopic scale.

Given the limited spatial resolution $(\sim 2.8 \mu \mathrm{m})$ of the reconstructed orientation maps, micron and sub-micron structures cannot be directly observed or examined. However, if the dislocation structure is coarse enough, the high orientation accuracy of the nf-HEDM measurements can potentially provide better information on orientation gradients, when compared to other orientation mapping techniques such as EBSD. Therefore, microstructure metrics such as intra-granular misorientation (IGM) and kernel average misorientation (KAM) fields (whose definitions are given below) are used to quantify orientation variations (within the length scale of the measurement) for individual grains in the microstructure. KAM can be used to quantify lattice imperfections locally, while IGM can be seen as a parameter that quantifies a long-range order/disorder in terms of crystallographic orientation within a single grain. Dislocation (mainly geometrically necessary dislocation (GND)) structures are associated with local orientation gradient and lattice mismatch; therefore, IGM and KAM maps can be associated with GND density or amount of plastic flow under deformation (Calcagnotto et al., 2010; Wright et al., 2011).

Intra-granular misorientation, or $\operatorname{IGM}(\mathbf{x})=\theta\left(g(\mathbf{x}), g_{\text {grain average }}\right)$, values are calculated for each voxel within a grain as the minimum misorientation angle $(\theta)$ between the orientation of each voxel $(g(\mathbf{x}))$ and the grain's average orientation ( $g_{\text {grain average }}$ ) accounting for all crystal symmetries. KAM is defined as the average misorientation angle between each voxel and its 26 nearest neighbors (in 3D). At each voxel, misorientation is computed as $K A M(\mathbf{x})=\left\langle\theta\left(g(\mathbf{x}), g_{\text {neighbor }}\right) \forall \theta<\psi\right\rangle$, where values above a threshold angle $\psi=5^{\circ}$ are excluded from the averaging in order to suppress contributions from high angle grain boundaries. Fig. 7(a-b) shows distributions of IGM and KAM in S2 and S3 states generated from the entire measured volumes of material. Both long range (IGM) and short range (KAM) orientations increase in mean value, in the standard deviation, and in the relative standard deviation. The fact that the standard deviation is comparable to the mean suggests that the deformation is highly heterogeneous. The broadening of the distributions due to plastic deformation in $\mathrm{Cu}$ is consistent with EBSD results reported previously in Lebensohn et al. (2008).

The overall effect of deformation is shown qualitatively in Fig. 8(a) by plotting the orientation fields of matched 2D cross-sections. Fig. 8(b) \& 8(c) show IGM and KAM maps of the matched layers shown in Fig. 8(a) 
for three macroscopic strains. Misorientation angles from IGM or KAM calculations are mapped to RGB colors at each point of the microstructure. In the fully annealed state, it is reasonable to consider that the orientation within each individual crystallite is uniform, to within the measurement noise. In this case, the IGM values are mostly zero throughout the map. On the other hand, the post-deformation grains show that each material point in a grain deviates in lattice orientation from the average. Note that expanded scale with $1.2^{\circ}$ maximum IGM is used to see the details in majority of the grain. Additionally, grain average calculations are done in $3 \mathrm{D}$, so in some cases, entire grain cross-sections have finite (even uniform) deviations from the average.

Similarly, KAM values at individual points were computed, where the expanded scale with the KAM ranging from 0 to 1 degree was used to plot the spatial maps as shown in Fig. 8(c). The spatial distribution of KAM in the deformed states illustrates large local orientation fluctuations in some grains, and less so in others. This clearly demonstrates the inhomogeneous nature of the plastic deformation and the importance of lattice orientation, grain size and shape, and local microstructural neighborhoods. We probe correlations with a variety of these parameters below.

\subsection{Microstructure Evolution at the Grain Scale}

Individual grains in a polycrystal are known (Poulsen, 2012) to exhibit heterogeneous and anisotropic deformation behavior. Grains are irregularly shaped and deformation is non-uniform throughout the microstructure. The variability in the change in orientation, size, and shape after straining could be attributed to various factors. Some grains are more favorably oriented to deform along the loading direction; therefore, they exhibit larger stretch while others show very little or no shape change after deformation. Additionally, initial shape and size as well as the orientations and the influence of the surrounding grains further enhance the anisotropic deformation of individual grains.

In order to illustrate the variety of deformation responses and the wealth of infomation that can be extracted from spatially resolved measurements, six interior grains spanning a wide range of orientations were selected and are shown in Fig. 9. Inverse pole figures for these grains are shown in Fig. 10 where the tail and head of the arrow represent the average orientation in S0 and S3 states, respectively.

To visualize the grain scale evolution, the IGM and KAM maps of the six selected grains are plotted in Fig. 9, at three different strain states. The grains are numbered in a descending order with respect to their initial volume, where grain \#1 is the largest, containing 129,215 voxels ( 1 voxel $=2.8 \times 2.8 \times 4 \mu^{3}$ ) and grain \#6 is the smallest containing 10,961 voxels. Statistics for the six grains are presented in Table 2, from which it can be seen that the grain shape change with deformation is very anisotropic, resulting in either monotonic increase in volume in some cases, for example grain \#1 and grain \#4 or monotonic decrease in volume as observed in grain \#6 or first increase then decrease and vice versa in grains \#2, \#3, and \#5. We note that volume increase or decrease of an individual grain corresponds to a change in the number of voxels whose orientation is within or exceeds the $5^{\circ}$ misorientation threshold chosen for grain segmentation, respectively. Therefore a local increase of one grain's volume corresponds to an equal decrease in the volume of its immediate neighbors and vice versa and the overall volume of the sample is conserved.

The irregular shapes of grains \#1 and \#6 at the initial state and the presence of gaps indicate that both grains have large numbers of neighbors some of which are twin-related. Although no correlation between shape change and initial orientation can be drawn from this particular analysis, the heterogeneous plastic deformation at the scale of individual grains is evident. For the six selected grains, an apparent strong dependence of magnitude of lattice rotation on initial orientation is observed in Fig. 10 and Table 2. This is in agreement with the findings in the literature (Pokharel et al., 2014; Oddershede et al., 2011; Winther et al., 2004). Grains 2 through 4 are located along the transition line ([110]-[112]) (Dillamore et al., 1972) and show a larger re-orientation than grains \#1 and \#5, which are closer to the stable orientations ([111] and [001]) and show smaller rotation. On the other hand, smaller orientation change is observed for grain \#6 presumably owing to its small size even though the grain is located along the transition line, where it would be expected to exhibit large reorientation. The heterogeneous spatial distributions of IGM and KAM values for individual grains are evident from Fig. 9. In addition, the $\langle I G M\rangle$ and the $\langle K A M\rangle$ values given in Table 2 show positive correlation between the grain average misorientation development and the grain size. A detailed statistical analysis based on large numbers of grains is presented in the following section to more deeply examine these correlations.

\subsection{Grain Fragmentation}

The segmentation of an orientation field into grains involves choosing a threshold misorientation angle, and the choice of the threshold angle is expected to determine the final grain size in a microstructure. During plastic deformation, some grains tend to subdivide into regions of high and low defect content, where sub-regions are 
separated by low angle boundaries. This phenomenon is termed grain fragmentation, which is commonly observed in plastic deformation to large strains (Quey et al., 2012), but for which no quantitative model exists.

Choosing a low threshold angle to segment the grains in a deformed state results in categorizing each subgrain region as an individual grain, which reduces the average grain size of the entire microstructure. The probability plots for the distribution of grain sizes at different threshold values and different strains were reported in (Pokharel, 2013), which exhibited little or no effect of the choice of threshold on the general shape of the grain size distribution. In this paper, we have examined the average grain sizes resulting from 4 different choices of threshold angle at three different strain states. Fig. 11 shows the average grain size versus misorientation threshold angle at S0, $\mathrm{S} 2$ and S3 states. From the plot, it is evident that the average grain size increases with increasing threshold values, and as expected, the deformed material shows a lower average grain size for a given threshold value.

\subsection{Effect of Grain Size on Defect Accumulation}

At a sub-micron length scale, mechanical properties of polycrystalline metals are known to be influenced by grain size distribution (Carroll et al., 2012). In this work, we have studied the origin of this dependency by examining the relationship between grain size and defect accumulation.

A correlation plot of the effect of grain size on defect concentration is presented in Fig. 12. Red points in Figs. 12(a) and 12(b) show the grain average IGM and KAM for each grain over the entire range of grain sizes. For both quantities, large variations are observed along with clustering of points displaying a positive trend, which is further confirmed by the trend line drawn through the average values. To better visualize the trend, grains are binned by grain size into eight equal bins, where the bin width is calculated as $b_{\text {width }}=\left(d_{\min }+d_{\max }\right) / n_{\text {bin }}$, where $d_{\min }$ and $d_{\max }$ are the minimum and the maximum grain size. Finally, misorientation angles falling in each bin are determined and averaged, and one standard deviation from the average is plotted for each bin. Clearly, defect accumulation at both long and short length scales, increases with grain size. In Fig. 12(c) the average IGM and KAM values are plotted; this shows that IGM has a stronger dependence on grains size than does KAM. This is not surprising since IGM is a measure of long-range orientation variation, which might intuitively be expected to increase, on average, with grain size, whereas KAM pertains to local variation. This observed influence of grain size is consistent with previous reports (Kamaya et al., 2005; Scheriau and Pippan, 2008). Furthermore, Valek et al. (2003) reported the microdiffraction measurements of plastic deformation on $\mathrm{Al}(\mathrm{Cu})$ inter-connect, where diffraction peaks from 4 neighboring grains were examined. Large streaking and rotation of diffraction peaks were observed for the largest grain, while smaller grains exhibited minimum peak distortion. This shape change was associated to the amount of defect content within a grain; thus, further confirming the dependence of plastic deformation on grain size.

Fig. 13 shows the grain average orientation for all 4930 tracked grains, colored by the $\langle I G M\rangle$ value at the S3 state. In Fig. 13(a), the grain size dependence is illustrated, where the marker (circle) size is normalized by the grain volume. From visual inspection, an interesting trend can be observed in the center of the triangle where most of the largest grains are located. It is this region where the grain orientation gives a lower Taylor factor with respect to the tensile loading axis. In addition, most grains in this region also seem to have smaller $\langle I G M\rangle$ values. Although Fig. 12(a), shows an average trend of increasing IGM with grain size, the few largest grains with low IGM values seen in Fig. 13(a) may be associated to the outliers visible in Fig. 12(a). The same information as shown in Fig. 13(a) is presented in Fig 13(b), this time varying the marker size and coloring by its $\langle I G M\rangle$ value, and it can be seen that grains that have moved or are moving towards the $\langle 001\rangle$ and $\langle 111\rangle$ corners develop larger intragranular misorientation, indicating defect accumulation, than the rest of the grains.

\subsection{Plastic Deformation Evolution}

During plastic deformation, some grains rotate as a unit, while others accumulate defects in the grain interior or near boundaries as above. Multiple slip allows a grain to accommodate an imposed strain, and heterogeneities in slip allow gradients to develop. The formation of overall deformation texture, as shown by the inverse pole figures in Fig.6(b-c), although not particularly sharp, illustrate the effect of plastic deformation. Additionally, Fig. 11 demonstrates grain fragmentation, which is in accord with results in the literature on subgrain structure formation within previously annealed grains (Mishra et al., 2005). Previous work on plastic deformation has also explored the effect of initial grain size on lattice rotation (Winther et al., 2004), and a similar correlation is hinted at by the lattice rotation plot for the six grains in Fig. 10.

Out of the 4930 tracked 3D grains, statistical analyses were performed on 1366 grains. Only grains that retained at least $60 \%$ of their initial volume are included so as to exclude fragmented grains, which would otherwise influence the lattice rotation calculation. Fig. 14 presents different crystallographic parameters for the filtered grains and illustrates the correlation between S2 and S3 states. From the grain size plot in Fig. 14(a), we see that the volume of the chosen grains are similar at the two deformed levels, which was as intended. Correlation 
for KAM and IGM are displayed in Figs. 14(b) \& 14(c), respectively, where the increase in both quantities after deformation is demonstrated. However, the spread in the data indicates an inhomogeneous accumulation with strain. A similar trend is seen for lattice rotation, where variability in the data points suggests the heterogenous nature of deformation.

In Fig. 15, grain reorientation is illustrated by plotting the grain average orientations at pre- and post-deformed states. The coloring scheme is based on the lattice rotation angle at S2 and S3 states. As shown in Fig. 15(a), in $\mathrm{S} 2$ state, grains in the center of the inverse pole figure seem to rotate more, while little or no rotation is observed in the rest of the grains. With further deformation, however, almost all grains exhibit some rotation at the S3 state, as seen in Fig. 15(c). It is also interesting to note that some grains in S2, with large average deviation from S0, seem to have re-arranged themselves such that their average orientation in S3 state is closer to their initial state orientation. For better visualization, grains with rotation angle larger than 2 degrees are shown in Figs. 15(b) and 15(d). Especially in S2 state, grains near the transition line exhibit the possibility to either rotate towards $\langle 001\rangle$ or along $\langle 111\rangle$, thus increasing the likelihood to develop long-range orientation gradients and promote sub-grain formation. However, only weak trend is observed in S3.

\subsection{Relationship Between Microstructural Features and Defect Accumulation}

Interfaces can be considered as sites with large geometrically necessary dislocation (GND) concentrations. Under quasi-static conditions, dislocation motion is the primary mechanism for plastic deformation in single phase $\mathrm{Cu}$. Damage in the form of cracks or voids may be related to concentrated slip, or high stress triaxiality, or large tractions across boundaries, depending on the circumstances. Owing to the difference in crystallographic orientations of adjacent grains, stress gradients tend to be large at the interfaces (Rollett et al., 2012). In addition, differences in Schmid and Taylor factors, owing to the initial orientations of the adjacent grains, result in large stress gradients (Pokharel et al., 2012) that lead to greater variation in slip rates on the various slip systems near grain boundaries and triple junctions. Thus, it is reasonable to expect that, in the absence of brittle or de-cohering particles, interfaces provide failure initiation sites. It is important to note again that the spatial resolution of the HEDM measurement is too coarse to resolve the dislocation cell structures that develop in $\mathrm{Cu}$. In order to separate regions of high and low defect density we use the magnitude of local orientation fluctuations, represented by the KAM calculation. This yields a consistent trend that would ideally be obtained from a submicron GND calculation (Calcagnotto et al., 2010).

In Fig. 16 we plot histograms of KAM values as a function of distance from the nearest grain boundaries. A Euclidean distance from the nearest boundary (Rollett et al., 2012) is computed for each voxel, the distances are binned and average KAM values are computed for each bin. Histograms for S2 and S3 are shown in Fig. 16(a) and 16(b), respectively. While there is clearly a wide range of variation at all distances, the highest values are observed predominately near boundaries. Fig. 16(b) clearly shows increasing orientation noise in the S3 state.

In 16(c), KAM values are first binned coarsely by grain size and then the associated points are binned by distance from grain boundaries. Five different grain size bins are plotted and in each bin the correlation between KAM and distance is displayed in solid black for S2 and gray for S3. Examination of the individual grain size bins shows that at both strain states, KAM values are larger near the boundary than in the interior. Detailed distributions are shown for three of the five size bins. Plotting the full data range illustrates the defect accumulation trend as a function of grain size. It is clear that the drop in defect content is more rapid in small grains (graphs labeled "(i)"), especially at S2 state, as schematically shown in Fig. 17. This might be because of the requirement to expend more energy to create dislocations in smaller grains than in larger grains. However, at large strains such as S3, the defect concentration in the grain interiors increases significantly irrespective of the grain size.

\section{Discussion}

Based on the spatially resolved orientation field data, local variations in plastic deformation were well captured throughout the microstructure evolution demonstrated by IGM and KAM plots shown in Figs. 8 and 9. Large orientation fluctuations were evident through both the spatial maps as well as correlation plots, particularly Fig. 16 , where the anisotropic plastic deformation is evident from the broad range of KAM values distributed near the interface. This is strong evidence that microstructural features along with local neighborhood exert a significant influence on defect localization (Rollett et al., 2010; Carroll et al., 2012), eventually leading to damage initiation and failure (Fensin et al., 2014).

Variation in the combination of active slip systems within a grain results in orientation gradients (Rollett et al., 2012), where the type of deformation microstructures depend on the initial orientation and grain size (Kalidindi et al., 2004). The analyses presented here, especially Figs 9-16, have confirmed the heterogeneous nature of plastic deformation and illustrated its dependence on various crystallographic parameters such as grain size and 
orientation. Grain averaging techniques (Poulsen et al., 2005; Bernier et al., 2011; Margulies et al., 2001) do not capture such plastic heterogeneities observed at a grain scale, which has been made possible through the spatially resolved orientation measurement (Hefferan et al., 2012; Li et al., 2012). Moreover, the current work offers a basis for studying the onset of plastic deformation, where the nf-HEDM technique combined with micro-tomography enable mapping of microstructure evolution at the stage where necking or void nucleation occur (Bingert et al., 2014); the latter represents the initiation of damage that leads eventually to ductile failure. Additionally, performing studies with other complementary X-ray based techniques to obtain spatial and time resolved elastic strains would be extremely beneficial in determining the stress variations within individual grains and would afford information on all active slip systems during deformation (Miller et al., 2012; Barton and Bernier, 2012). It is likely that the modeling community will benefit greatly from such measurements, as invaluable information on grain evolution, local orientation and strain change, and neighborhood effects can be exploited for further validating, developing and improving the deformation models (Wen et al., 2013). Access to such data would directly enhance the predictive capability of the current plasticity simulations (Groeber et al., 2008), which still heavily rely on 3D synthetic structures and statistical data as input to the models (Lee et al., 2011; Lebensohn and Pokharel, 2014).

The success of the nf-HEDM technique to non-destructively measure and map spatially resolved statistically significant bulk 3D grain orientations through multiple stages of strain steps has been a major breakthrough in advancing the capability of studying microstructure evolution under deformation. Considering that a single sample was measured in-situ at multiple strain states, as illustrated in Figs. 2 and 3, it is encouraging that dynamic studies under varying loading condition will be a possibility in the near future.

The experiment reported here is novel in that the observation and analysis of statistically significant bulk 3D grain evolution and mapping full local neighborhoods of individual grains has never been done before. However, it is important to bear in mind that, notwithstanding the advance in capability, it is only a step towards solving a very complicated problem of understanding the full mechanics of the deformation process. Further challenges will exist before this type of information can be applied to, for example, designing new materials.

\section{Conclusions}

A nf-HEDM experiment was performed and bulk 3D volumes of a $\mathrm{Cu}$ sample undergoing uniaxial strain were measured at different stages of deformation. For each state, large data sets were obtained with $\sim 7000$ grains, which allowed for representative sampling of the defect accumulation and plastic deformation. Selected features and their evolution were extracted from the large data sets. The developed tools were tested, and the analyses were performed on the initial, $6 \%$ and $12 \%$ deformed microstructures. Microstructural evolution was studied at both the macroscopic and local levels.

The availability of large statistical data volumes indicated that the mechanisms involved in heterogeneous deformation are more complex than the way they are currently being described. This has posed several challenges in terms of data handling, mining and representation of various crystallographic parameters, and an ongoing effort is aimed at developing quantitative tools and appropriate metrics for extracting relevant information that would give broader insights on the physical mechanisms involved in the deformation process. Along with the quantitative representation of the extracted information from the experimental data presented here, comparisons with numerical modeling based on crystal plasticity will follow.

In summary, our results emphasize that deformation in copper is dominated by heterogeneity. While our results are in agreement with macroscopic observations such as texture evolution, distributions of multiple quantities related to defect content within individual grains show standard deviations of the same order as average values. The breadth of these distributions is presumably due to the fact that each grain experiences a unique anisotropic environment due to its specific neighborhood. Within these broad distributions, we observe a trend toward increased disorder in proximity to grain boundaries as is consistent with dislocation pile ups. These data raise the question as to whether computational plasticity models based on standard constitutive relations can be tuned to track the observed heterogeneity. As is shown in a recent review (Pokharel et al., 2014), such efforts to date have not been successful.

It is only with the voxelized, forward model based reconstruction method applied to near-field HEDM data sets that the characterizations presented here become possible. Future work can be expected to yield even more comprehensive data sets with spatially resolved elastic strain information to complement the orientation maps presented here. Advances in data collection technologies, in-situ sample environments, and analysis methods will combine to yield data sets that can be used to test and constrain computational models. 


\section{Acknowledgements}

This research was supported by the US Department of Energy, Office of Basic Energy Sciences, Division of Materials Sciences and Engineering, under award DESC0002001. Use of the Advanced Photon Source was supported by the US Department of Energy, Office of Science, Office of Basic Energy Sciences, under contract No. DE-AC02-06CH11357. Partial support from Los Alamos National Laboratory's Directed Research and Development (LDRD-DR Project 20140114DR) is also acknowledged. Part of this work was performed under the auspices of the U.S. Department of Energy by Lawrence Livermore National Laboratory under Contract DEAC52-07NA27344. This Research was also supported in part by the National Science Foundation through XSEDE resources provided by Texas Advanced Computing Center under grant number DMR080072, and in part by the National Energy Research Scientific Computing Center, which is supported by the Office of Science of the U.S. Department of Energy under Contract No. DE-AC02-05CH11231.

\section{References}

Barabash, R., Ice, G., Liu, W., Barabash, O., 2009. Polychromatic microdiffraction characterization of defect gradients in severely deformed materials. Micron 40 (1), 28-36.

Barton, N., Bernier, J., 2012. A method for intragranular orientation and lattice strain distribution determination. Journal of Applied Crystallography 45 (6), 1145-1155.

Bernier, J., Barton, N., Lienert, U., Miller, M., 2011. Far-field high-energy diffraction microscopy: a tool for intergranular orientation and strain analysis. The Journal of Strain Analysis for Engineering Design 46 (7), 527-547.

Bernier, J., Miller, M., 2006. A direct method for the determination of the mean orientation-dependent elastic strains and stresses in polycrystalline materials from strain pole figures. Journal of applied crystallography 39 (3), 358-368.

Bingert, J., Suter, R., Lind, J., Li, S., Pokharel, R., Trujillo, C., 2014. High-energy diffraction microscopy characterization of spall damage. In: Dynamic Behavior of Materials, Volume 1. Springer, pp. 397-403.

Calcagnotto, M., Ponge, D., Demir, E., Raabe, D., 2010. Orientation gradients and geometrically necessary dislocations in ultrafine grained dual-phase steels studied by 2d and 3d ebsd. Materials Science and Engineering: A 527 (10), 2738-2746.

Carroll, J., Brewer, L., Battaile, C., Boyce, B., Emery, J., 2012. The effect of grain size on local deformation near a void-like stress concentration. International Journal of Plasticity 39, 46-60.

Cerreta, E., Bingert, J., Gray III, G., Trujillo, C., Lopez, M., Bronkhorst, C., Hansen, B., 2013. Microstructural examination of quasi-static and dynamic shear in high-purity iron. International Journal of Plasticity 40, 23-38.

Dillamore, I., Morris, P., Smith, C., Hutchinson, W., 1972. Transition bands and recrystallization in metals. Proceedings of the Royal Society of London. A. Mathematical and Physical Sciences 329 (1579), 405-420.

Fensin, S., Escobedo-Diaz, J., Brandl, C., Cerreta, E., Gray III, G., Germann, T., Valone, S., 2014. Effect of loading direction on grain boundary failure under shock loading. Acta materialia 64 (0), 113-122.

Groeber, M., Ghosh, S., Uchic, M., Dimiduk, D., 2008. A framework for automated analysis and simulation of 3d polycrystalline microstructures.: Part 1: Statistical characterization. Acta Materialia 56 (6), 1257-1273.

Hefferan, C., Lind, J., Li, S., Lienert, U., Rollett, A., Suter, R., 2012. Observation of recovery and recrystallization in high-purity aluminum measured with forward modeling analysis of high-energy diffraction microscopy. Acta Materialia 60 (10), $4311-4318$.

Joffe, A., Kirpitcheva, M., 1922. Rontgenograms of strained crystals. Philosophical Magazine 43 (253), 204-206, 6TH SERIES.

Kalidindi, S., Bhattacharyya, A., Doherty, R., 2004. Detailed analyses of grain-scale plastic deformation in columnar polycrystalline aluminium using orientation image mapping and crystal plasticity models. Proceedings of the Royal Society of London. Series A: Mathematical, Physical and Engineering Sciences 460 (2047), 1935-1956.

Kamaya, M., Wilkinson, A. J., Titchmarsh, J. M., 2005. Measurement of plastic strain of polycrystalline material by electron backscatter diffraction. Nuclear engineering and design 235 (6), 713-725.

Larson, B., Yang, W., Ice, G., Budai, J., Tischler, J., 2002. Three-dimensional x-ray structural microscopy with submicrometre resolution. Nature 415 (6874), 887-890.

Lebensohn, R., Brenner, R., Castelnau, O., Rollett, A., 2008. Orientation image-based micromechanical modelling of subgrain texture evolution in polycrystalline copper. Acta materialia 56 (15), 3914-3926.

Lebensohn, R., Pokharel, R., 2014. Interpretation of microstructural effects on porosity evolution using a combined dilatational/crystal plasticity computational approach. JOM 66 (3), 537-443.

Lee, S.-B., Lebensohn, R., Rollett, A., 2011. Modeling the viscoplastic micromechanical response of two-phase materials using fast fourier transforms. International Journal of Plasticity 27 (5), 707-727.

Li, S., Lind, J., Hefferan, C., Pokharel, R., Lienert, U., Rollett, A., Suter, R., 2012. Three-dimensional plastic response in polycrystalline copper via near-field high-energy x-ray diffraction microscopy. Journal of Applied Crystallography 45 (6), 1098-1108.

Li, S., Suter, R., 2013. Adaptive reconstruction method for three-dimensional orientation imaging. Journal of Applied Crystallography 46 (2), $512-524$.

Lienert, U., Li, S. F., Hefferan, C. M., Lind, J., Suter, R. M., Bernier, J. V., Barton, N. R., Brandes, M. C., Mills, M. J., Miller, M. P., Jakobsen, B., Pantleon, W., 2011. High-energy diffraction microscopy at the advanced photon source. JOM 63 (7), 70-77.

Lind, J., Li, S., Pokharel, R., Lienert, U., Rollett, A., Suter, R., 2014. Tensile twin nucleation events coupled to neighboring slip observed in 3d. Acta Materialia 76, 213-220.

Ludwig, W., Schmidt, S., Lauridsen, E., Poulsen, H., 2008. X-ray diffraction contrast tomography: a novel technique for three-dimensional grain mapping of polycrystals. i. direct beam case. Journal of Applied Crystallography 41 (2), 302-309.

Margulies, L., Winther, G., Poulsen, H., 2001. In situ measurement of grain rotation during deformation of polycrystals. Science 291 (5512), 2392-2394.

McDonald, J., Ma, S., Pollock, T., Yalisove, S., Nees, J., 2008. Femtosecond pulsed laser ablation dynamics and ablation morphology of nickel based superalloy cmsx-4. Journal of Applied Physics 103 (9), 093111.

Miller, M., Suter, R., Lienert, U., Beaudoin, A., Fontes, E., Almer, J., Schuren, J., 2012. High-energy needs and capabilities to study multiscale phenomena in crystalline materials. Synchrotron Radiation News 25 (6), 18-26. 
Mishra, A., Richard, V., Gregori, F., Asaro, R., Meyers, M., 2005. Microstructural evolution in copper processed by severe plastic deformation. Materials Science and Engineering: A 410, 290-298.

Oddershede, J., Schmidt, S., Poulsen, H., Margulies, L., Wright, J., Moscicki, M., Reimers, W., Winther, G., 2011. Grain-resolved elastic strains in deformed copper measured by three-dimensional x-ray diffraction. Materials characterization 62 (7), 651-660.

Pokharel, R., 2013. Spatially resolved in-situ study of plastic deformation in polycrystalline copper using high-energy x-rays and full-field simulations. Ph.D. thesis, Carnegie Mellon University.

Pokharel, R., Li, S., Lind, J., Hefferan, C., Lienert, U., Lebensohn, R., Suter, R., Rollett, A., 2012. Quantifying damage accumulation using state-of-the-art fft method. Materials Science Forum 702, 515-518.

Pokharel, R., Lind, J., Kanjarla, A., Li, S., Lebensohn, R., Kenesei, P., Suter, R., Rollett, A., 2014. Polycrystal plasticity: Comparison between grain scale observations of deformation and simulations. Annual Review of Condensed Matter Physics 5, 317-346.

Pouillier, E., Gourgues, A.-F., Tanguy, D., Busso, E. P., 2012. A study of intergranular fracture in an aluminium alloy due to hydrogen embrittlement. International Journal of Plasticity 34, 139-153.

Poulsen, H., 2012. An introduction to three-dimensional x-ray diffraction microscopy. Journal of Applied Crystallography 45 (6), $1084-1097$.

Poulsen, H., Jensen, D., Vaughan, G., 2004. Three-dimensional x-ray diffraction microscopy using high-energy x-rays. MRS bulletin 29 (03), $166-169$.

Poulsen, H., Lienert, U., Pantleon, W., 2005. Characterisation of orientation distributions of individual grains within deformed metals. Materials science and technology 21 (12), 1397-1400.

Quey, R., Dawson, P., Driver, J., 2012. Grain orientation fragmentation in hot-deformed aluminium: Experiment and simulation. Journal of the Mechanics and Physics of Solids 60 (3), 509-524.

Rollett, A., Lebensohn, R., Groeber, M., Choi, Y., Li, J., Rohrer, G., 2010. Stress hot spots in viscoplastic deformation of polycrystals. Modelling and Simulation in Materials Science and Engineering 18 (7), 074005.

Rollett, A., Wagner, F., Allain-Bonasso, N., Field, D., Lebensohn, R., 2012. Comparison of gradients in orientation and stress between experiment and simulation. Materials Science Forum 702-703, 463-468.

Rowenhorst, D., Lewis, A., 2011. Image processing and analysis of 3-d microscopy data. JOM 63 (3), 53-57.

Scheriau, S., Pippan, R., 2008. Influence of grain size on orientation changes during plastic deformation. Materials Science and Engineering: A 493 (1), 48-52.

Schwartz, A., Kumar, M., Adams, B., Field, D., 2009. Electron Backscatter Diffraction in Materials Science, 2nd Edition. Springer, New York.

Shade, P., Groeber, M., Schuren, J., Uchic, M., 2013. Experimental measurement of surface strains and local lattice rotations combined with $3 \mathrm{~d}$ microstructure reconstruction from deformed polycrystalline ensembles at the micro-scale. Integrating Materials and Manufacturing Innovation 2 (1), 5.

Shade, P. A., Groeber, M. A., Uchic, M. D., Turner, T. J., Dimiduk, D. M., Woodward, C., Choi, Y.-S., Wheeler, R., 2011. Micro-tensile testing and 3d-ebsd characterization of pure nickel multi-crystals (preprint). Tech. rep., DTIC Document.

Suter, R., Hennessy, D., Xiao, C., Lienert, U., 2006. Forward modeling method for microstructure reconstruction using x-ray diffraction microscopy: Single-crystal verification. Review of scientific instruments 77 (12), 123905.

Uchic, M., Groeber, M., Rollett, A., 2011. Automated serial sectioning methods for rapid collection of 3-d microstructure data. Jom 63 (3), 25-29.

Valek, B., Tamura, N., Spolenak, R., Caldwell, W., MacDowell, A., Celestre, R., Padmore, H., Bravman, J., Batterman, B., Nix, W., et al., 2003. Early stage of plastic deformation in thin films undergoing electromigration. Journal of applied physics 94 (6), $3757-3761$.

Warren, B., 1969. X-ray Diffraction. Courier Dover Publications.

Wen, W., M'Guil, S., Ahzi, S., Gracio, J., 2013. Coupled effects of the lattice rotation definition, twinning and interaction strength on the fcc rolling texture evolution using the viscoplastic $\phi$-model. International Journal of Plasticity 46, 23-36.

Winther, G., Margulies, L., Schmidt, S., Poulsen, H., 2004. Lattice rotations of individual bulk grains part ii: correlation with initial orientation and model comparison. Acta materialia 52 (10), 2863-2872.

Wright, S. I., Nowell, M. M., Field, D. P., 2011. A review of strain analysis using electron backscatter diffraction. Microscopy and microanalysis 17 (03), 316-329.

Zeghadi, A., N'guyen, F., Forest, S., Gourgues, A.-F., Bouaziz, O., 2007. Ensemble averaging stress-strain fields in polycrystalline aggregates with a constrained surface microstructure-part 1: Anisotropic elastic behaviour. Philosophical Magazine 87 (8-9), 1401-1424.

Zhao, Z., Ramesh, M., Raabe, D., Cuitino, A., Radovitzky, R., 2008. Investigation of three-dimensional aspects of grain-scale plastic surface deformation of an aluminum oligocrystal. International Journal of Plasticity 24 (12), 2278-2297. 


\section{Figure Captions}

Figure 1: Schematic of the experimental apparatus for nf-HEDM measurements under in-situ tensile loading. The sample is held inside a cylindrical stainless steel tube with an X-ray transparent Beryllium window section. The bottom end of the sample is fastened to a load cell which is mounted on a translation stage. As the stage is lowered, it exerts tensile force on the sample. The loading apparatus is in turn fastened to translation stages that are used to center the sample on the rotation axis of an air bearing rotation stage. The X-ray sensing element of the high resolution imaging detector system is positioned at $L=5$ to $15 \mathrm{~mm}$ downstream of the rotation axis. For each "layer measurement" images of diffraction patterns are collected as the sample is rotated about the indicated axis (referred to as $\omega$ ) through a succession of intervals of width $\delta \omega=1^{\circ}$ which span $180^{\circ}$. Two or three such image sets are collected at different detector positions, $L$, so as to encode the position of origin and the propagation direction of diffracted beams (Suter et al., 2006; Lienert et al., 2011).

Figure 2: Experimental stress-strain curve along with one layer of orientation and confidence maps from each of the five measured strain states. Load and displacement data are used to compute the true stress versus true strain curve shown by the red line. Square symbols show locations at which nf-HEDM measurements were taken; these extend from 0 to $21 \%$ tensile strain. The 2D maps plotted outside the stressstrain graph represent reconstructed orientation fields from each of the corresponding strain levels. Colors correspond to an RGB mapping of Rodrigues vector components specifying the local crystal orientation. These cross-sections are the top-most layers of the respective measured volumes and are not necessarily from the same region in the sample. The 2D maps plotted inside the stress-strain graph are the confidence, $C$, maps for the reconstructed orientation fields. In all the maps, black lines are drawn between nearest neighbor voxels that have $>5^{\circ}$ degree misorientation so as to outline grain boundaries. Note: High frequency noise in the stress-strain curve due to readout noise from the load cell.

Figure 3: 3D volumes of the reconstructed microstructures obtained from the forward modeling method. (a) S0, (b) S2, and (c) S3. The increasing number of layers measured (see Table 1) is evident. Coloring is by Rodrigues vector components as in Fig. 2.

Figure 4: Orientation maps showing a vertical planar section from the center of the 3D volumes at (a) S0, (b) S2, and (c) S3. The dotted white lines in (b) and (c) show the approximate region occupied by the material measured in S0. (d) Grain boundaries extracted from the three states to further illustrate the coincidence of the two deformed volumes with the initial measured microstructure.

Figure 5: Orientation maps for a single grain at S0 (left), S2 (center) and S3 (right). The average orientation of these voxels changes by $1.4^{\circ}$ and $2.7^{\circ}$ in passing from the initial to the deformed states; these small rotations do not produce distinguishable color changes on this scale which is normalized to span the entire cubic fundamental zone.

Figure 6: Inverse pole figures showing the distribution of crystal poles that are parallel to the laboratory sample (001) axis which is the macroscopic tensile axis. Left to right figures correspond to S0, S2 and S3 strain states. Colors show multiples of a random distribution for initial, $6 \%$ and $12 \%$ tensile strain states. The color scale is the same for each map and minimum and maximum populations are noted for each state.

Figure 7: Misorientation angle histograms of (a) IGM and (b) KAM for the two deformed states. The increase in the mean misorientation as well as the broadening of the curve at S3 indicate defect accumulation with strain. $\mu$ and $\sigma$ are the mean and standard deviation in degrees.

Figure 8: Registered layers for pre- and post-deformation states. (a) Orientation maps, (b) IGM maps, and (b) KAM maps for S0 (left), S2 (center) and S3 (right) tensile strain for the registered layers. Heterogeneous plastic deformation with orientation gradient hot spots can be observed in both S2 and S3 deformation states.

Figure 9: Images of six registered and tracked grains colored by IGM (left columns) and by KAM (right columns). Each quantity is shown in each grain for each reported strain state. In order to visualize both small and large grains, the spatial scale is different for each grain. Volumes are given in Table 2 . 
Figure 10: Lattice rotation between the S0 and S3 strain states for six selected bulk grains in Fig. 9. The gray arrows indicate the direction of rotation, starting at S0 and ending at S3 average orientations. The line connecting the two shapes is colored by magnitude of the rotation angle.

Figure 11: Average grain size versus the misorientation threshold used in grain segmentation for initial (S0), 6\% (S2), and 12\% (S3) states. Decrease in grain size with decreasing misorientation threshold and increasing strain is evident.

Figure 12: Statistical study of the effect of grain size on misorientation development at $12 \%$ strain. Grain average (a) IGM and (b) KAM versus grain size were calculated for 4930 grains. Each red dot represent a 3D grain with volume larger than 100 voxels. Data was binned and the black dotted line passes through the average of all the values falling in each bin and the error bar is the standard deviation. (c) Binned average IGM and KAM values are plotted with the same scale to illustrate the slope of the lines representing the two quantities. Clearly the IGM dependence on grain size is greater than that of KAM.

Figure 13: Final orientation for 4930 tracked grains after $12 \%$ tensile strain, where (a) the radius of the circle is determined by the grain size and colored by the grain averaged IGM angle. Same grains are illustrated in (b) where both the size and color of the open circles are varied by the IGM value.

Figure 14: Correlations between states S2 and S3 of different crystallographic parameters. Grain parameters plotted are (a) grain size as number of voxels, (b) average KAM, (c) average IGM, and (d) average rotation from S0 state after deformation. The dotted gray $y=n x$ lines, where $n=1,2,3 \ldots$, show how different grains evolve at the two strain states. As shown in (a) grain sizes are stable as expected for the selection criterion although larger relative changes occur for smaller grains. In (b-c), we see that most grains in S3 state seem to develop 2 to 4 times more KAM and IGM than in S2 state. In (d), we see that most of the rotation in S3 was between 0 and 2 times that of S2.

Figure 15: The trend in rotation and its dependence on initial orientation for 1366 tracked grains is shown, where the tail and head of the arrow indicate the initial and final orientations, respectively. The arrows are colored by rotation angles from(a-b) S0 to S2 and (c-d)S0 to S3 strain states. Despite the noise, the grains along the transition line clearly exhibit larger rotations (more obviously at the S2 state) in (a) than in (c). To clarify the trend in (a) and (c), grains are filtered such that the pair of plots in (b) and (d) only show grains with rotation greater than $2^{\circ}$.

Figure 16: In this figure, we examine KAM as a function of three major parameters: strain state, distance from grain boundary, and grain size. We start by displaying all KAM values for (a) S2 and (b) S3 states, partitioned into ten bins, plotting a point for each grain in each bin and also displaying the number of data points in each bin. From this data we clearly see a trend of decreasing KAM as a function of distance from grain boundary, and increase in KAM values in the interior of the grains in the larger strain state, S3. In order to study the grain size dependence of KAM, we further partition the data into five grain size bins in (c). In the large plot in the middle, we display the envelopes of the data for both states S2 and S3 and the five grain size bins together, for easier comparison. The smaller plots, labeled (i), (ii), and (iii), show the full data range in individual bins for three of the five grain size bins, in both S2 and S3 states. The grain size partitioned data is consistent in that the KAM values of the larger strain state, S3, are increased and have made their way further into the grain interiors. Furthermore, we see the additional feature that the KAM vs distance from grain boundary gradient is much larger in the smaller than in the larger grains, with the larger grains experiencing a much smoother, more uniform increase in KAM as a function of distance from grain boundary.

Figure 17: The data in Fig. 16(c) implies the possibility of the scenario depicted above, in which a dramatic difference in KAM versus distance to grain boundary is seen in two adjacent grains of different size. One possible explanation for this is that while the two grains experience a similar macroscopic strain near the interface, in smaller grains the defect content accumulation is mostly because of incompatibilities near the boundary as dislocation pile-up is harder in the grain interior. In the case of larger grains, dislocation pile up and dislocation motion are easier in response to shear stresses and, as a result, more uniform defect accumulation is observed in the grain interior and near boundaries. 


\section{Table Captions}


Copper Specimen $\quad$ Rotation Axis

Imaging Detector

Bragg Peak

Beryllium Window

$\checkmark$ Stainless Steel Tube
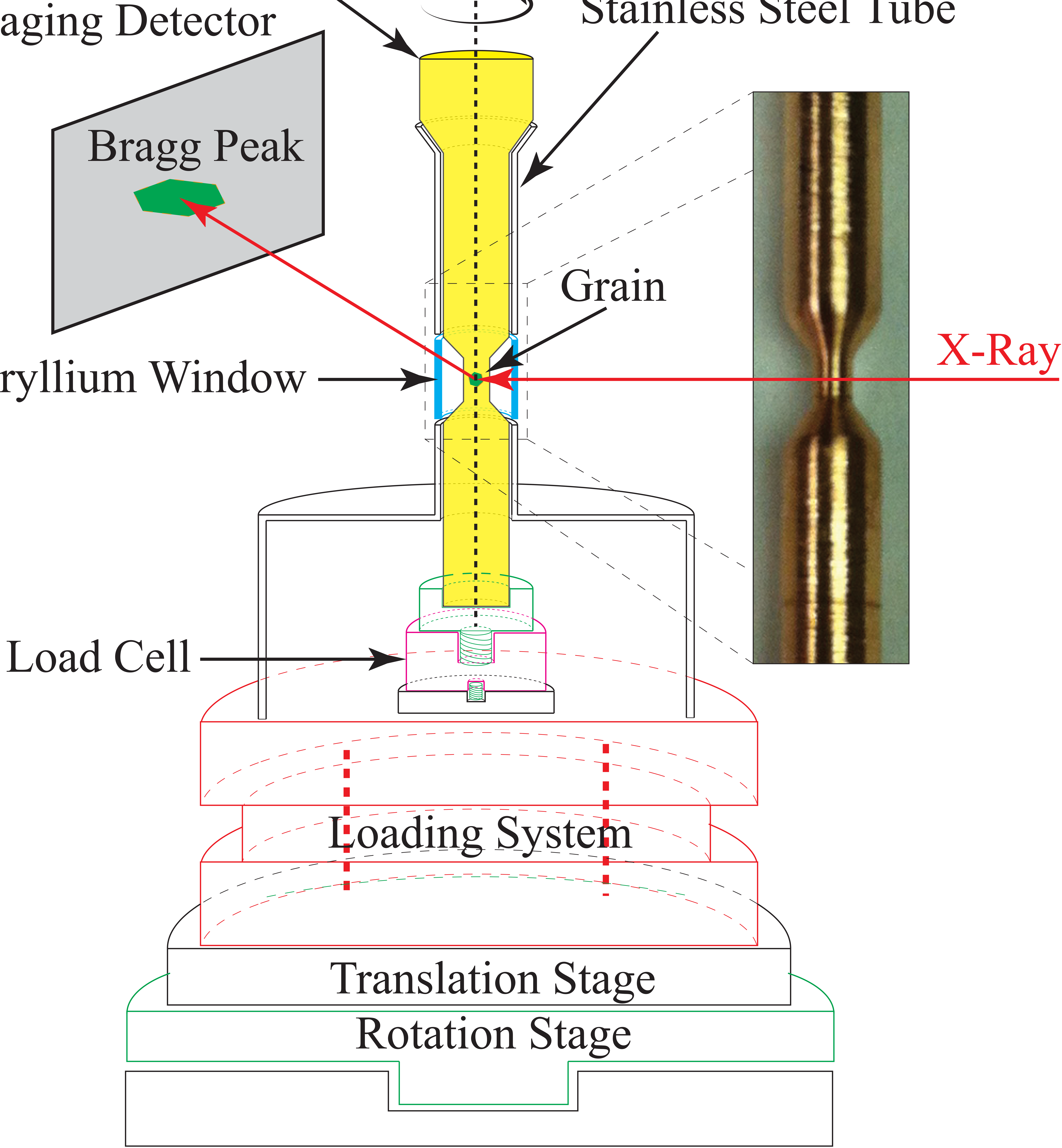



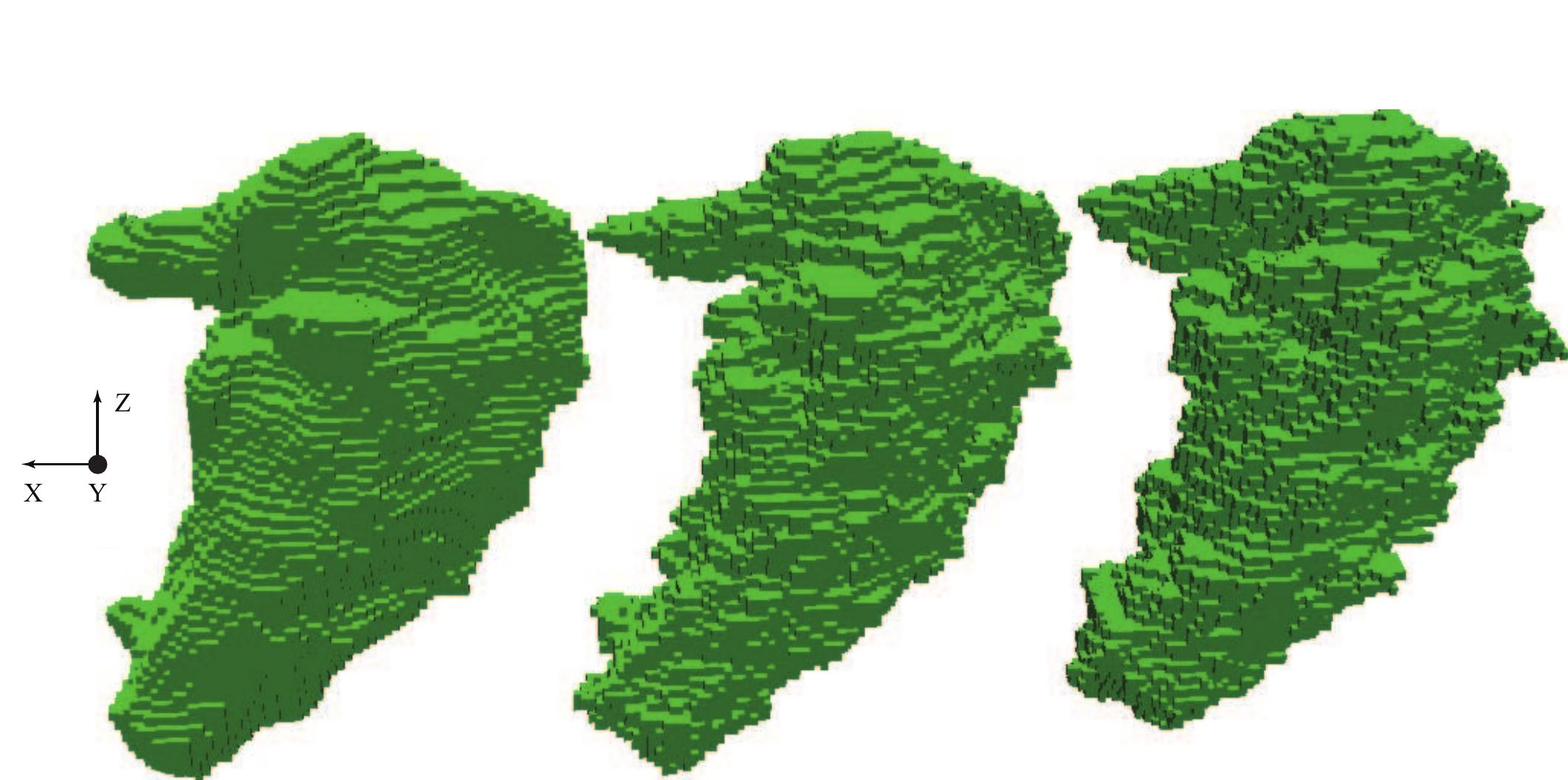

Figure 


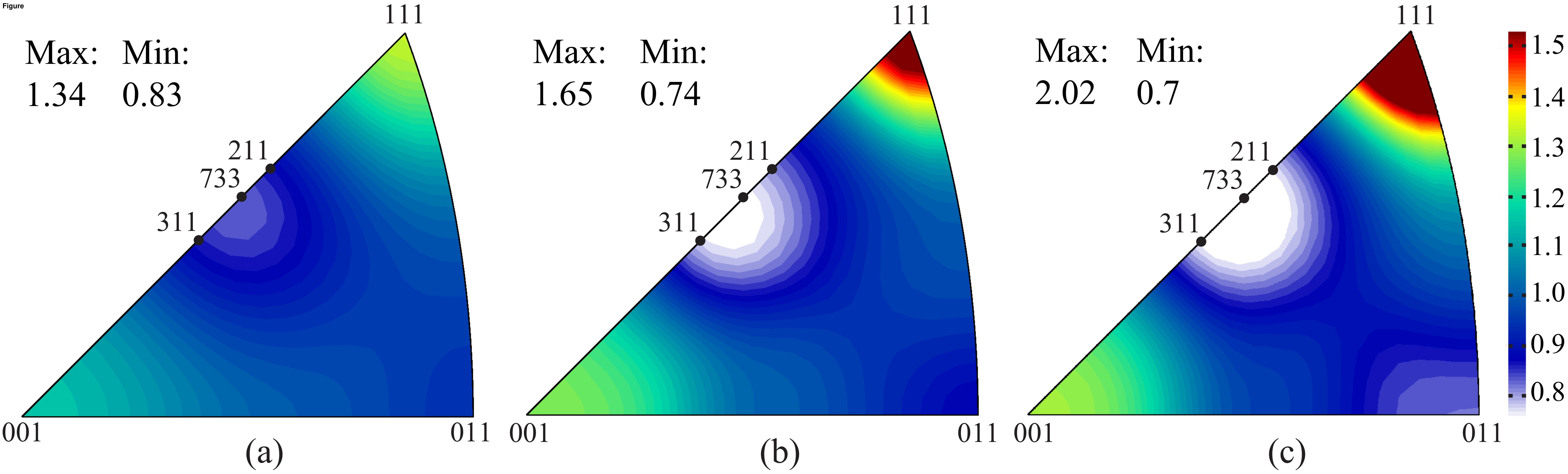




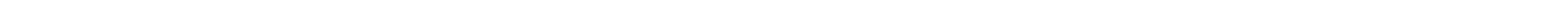


$\begin{array}{llll}0 \% & 6 \% & 12 \%\end{array}$
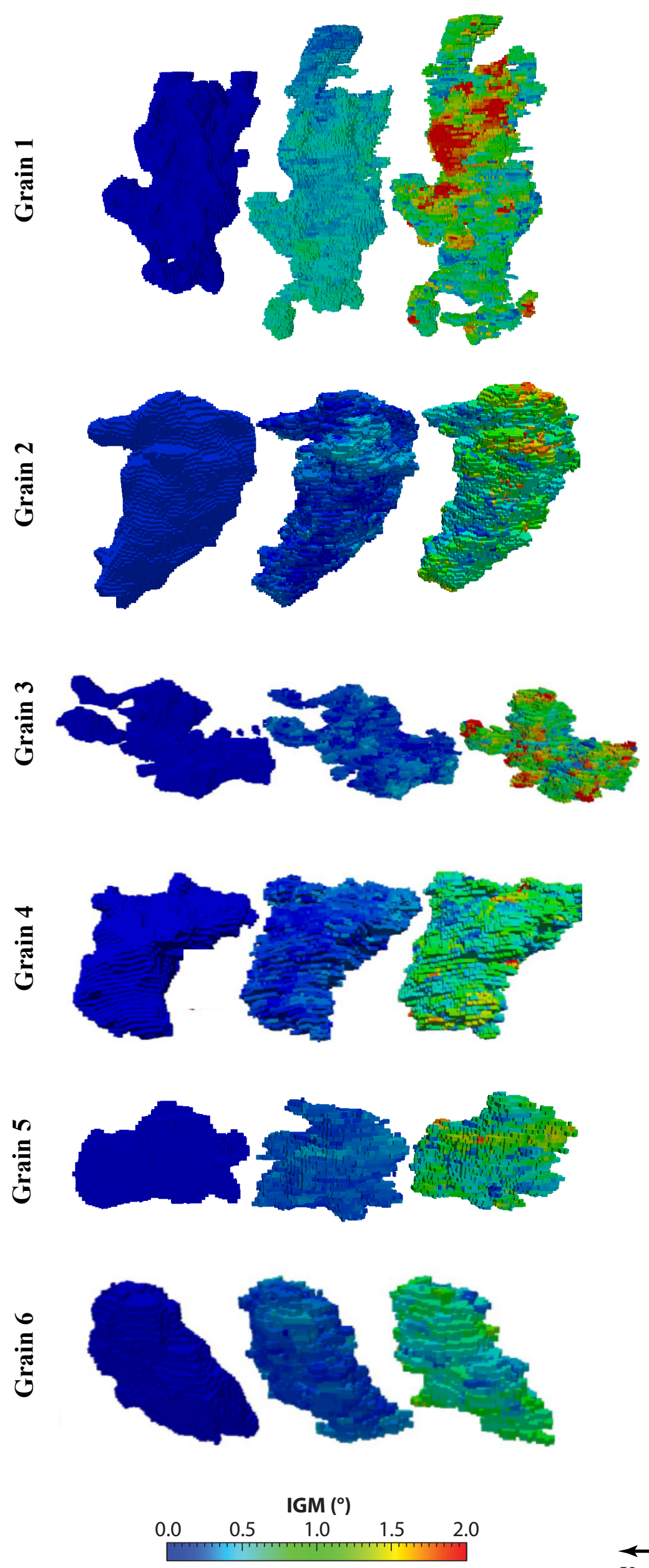

(a)
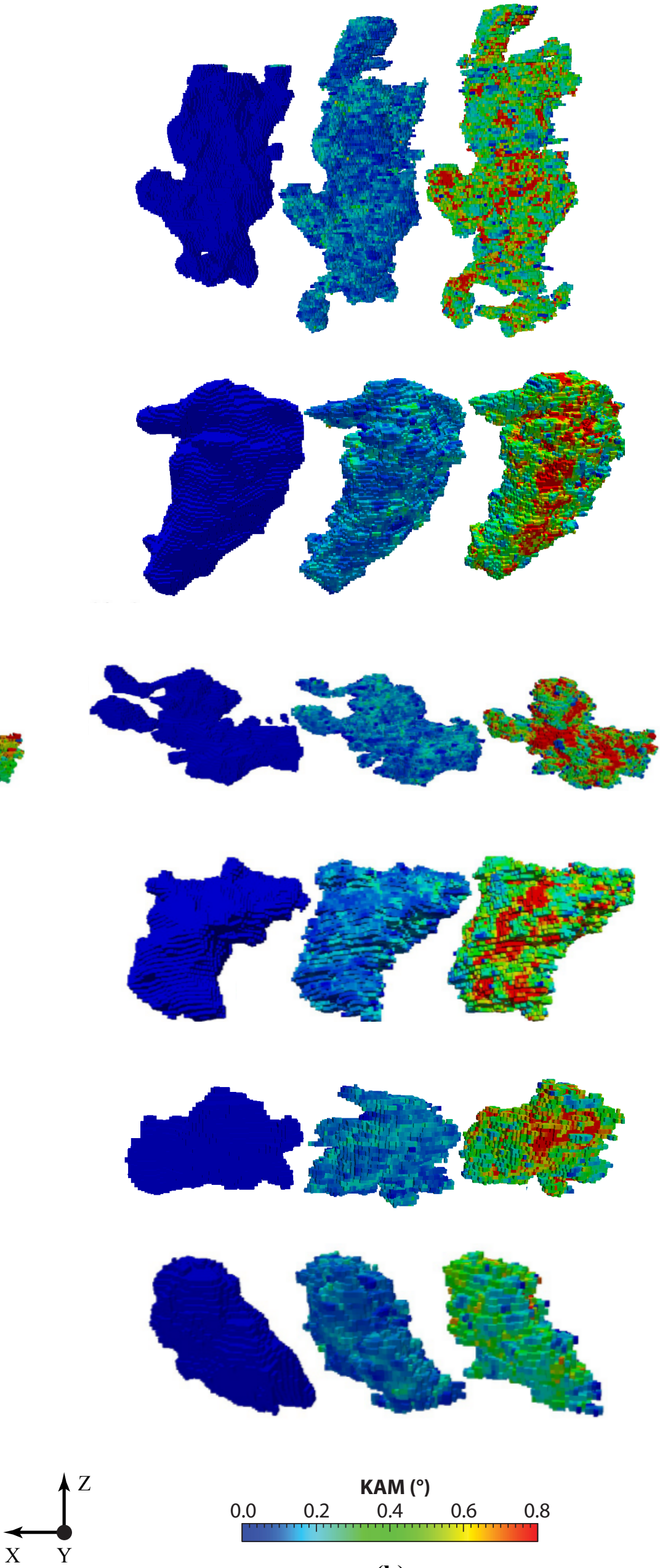

(b) 


\section{Figure}

\section{Rotation $\left({ }^{\circ}\right)$}

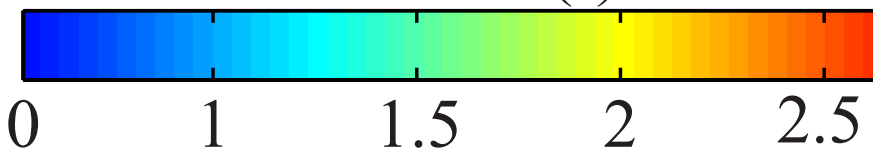

O so

$\square$ S3

Q1

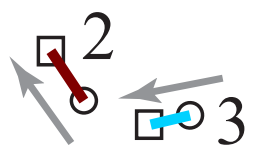

ㄷ4 
(avi)

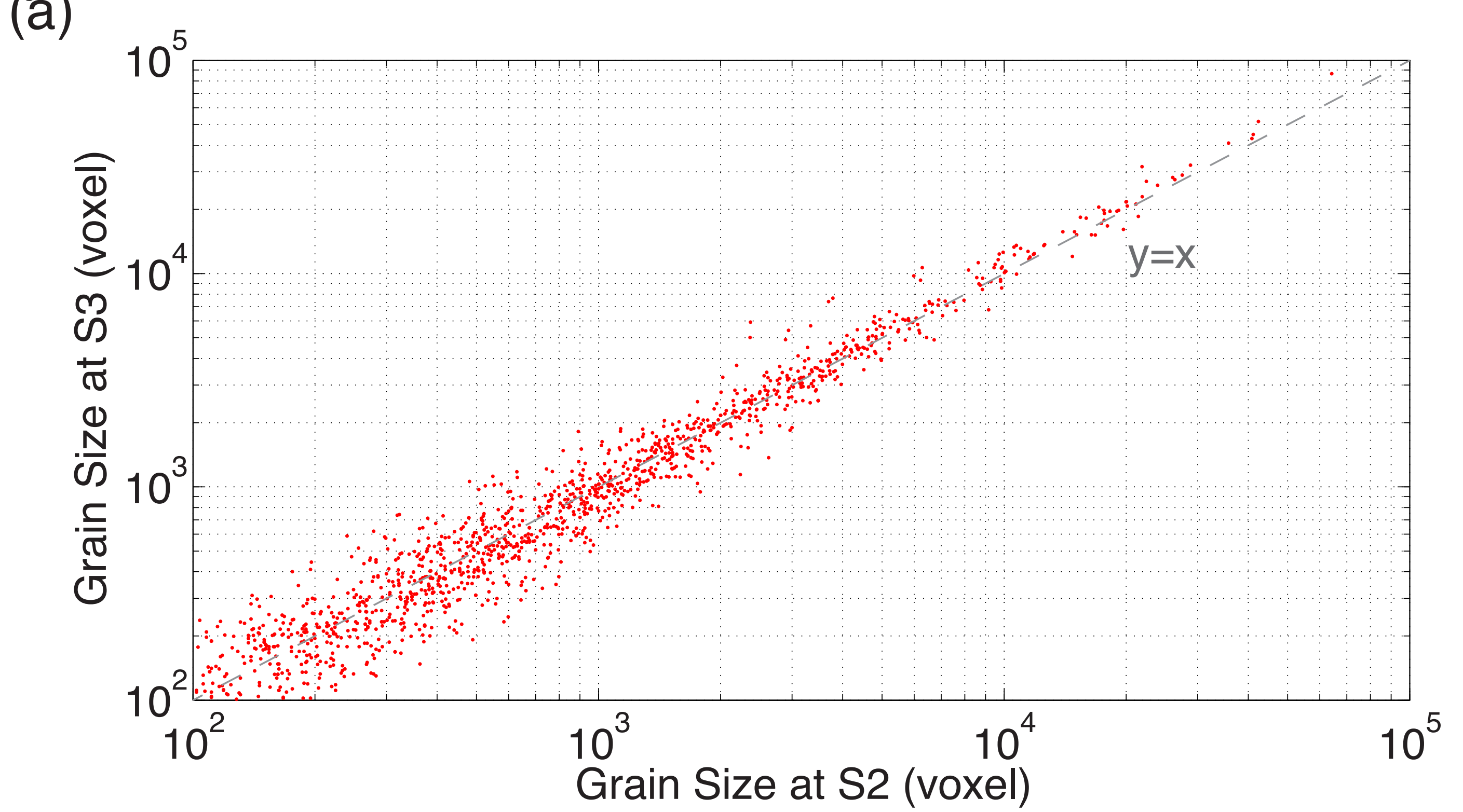

(c)

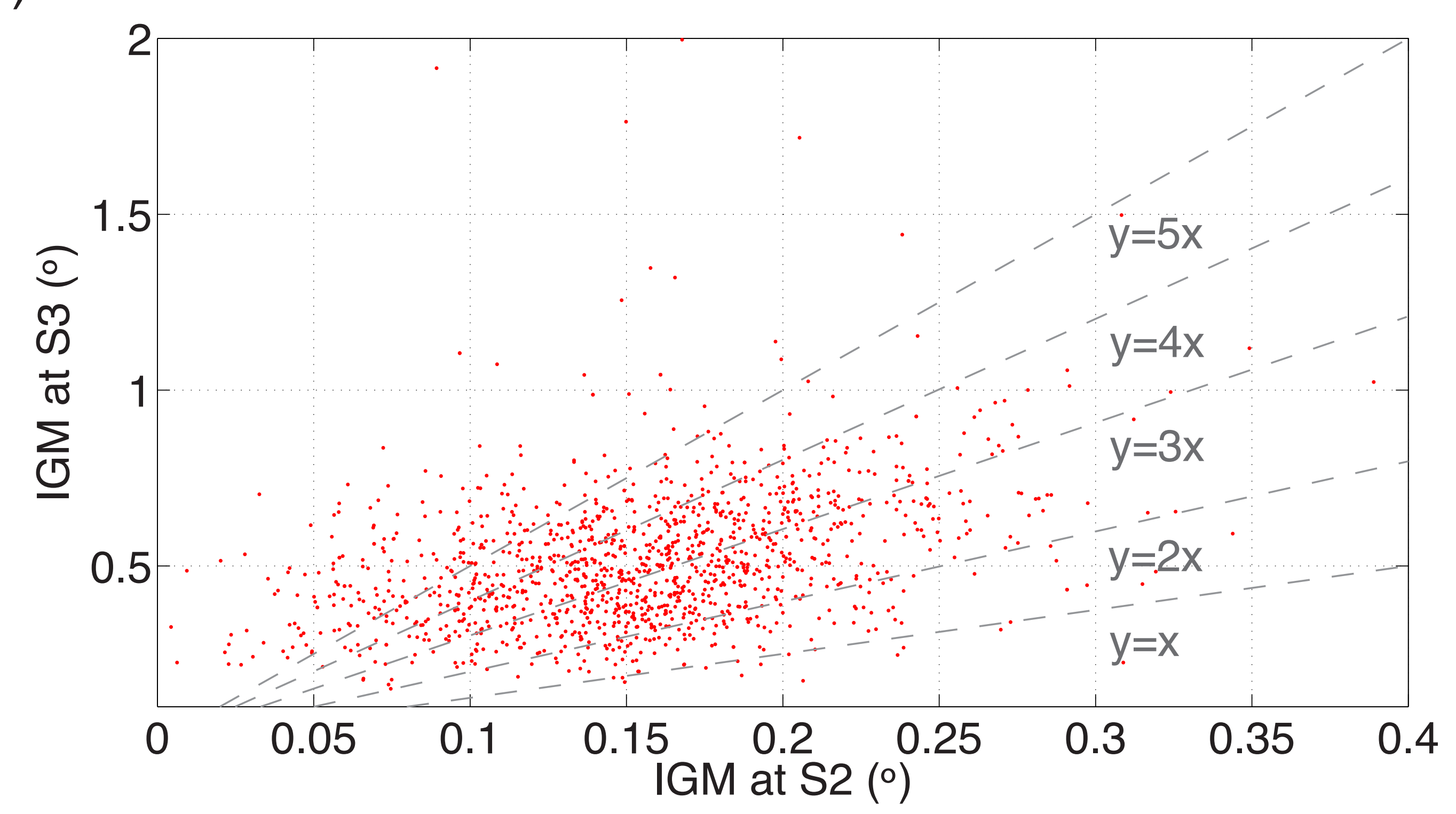

(b)

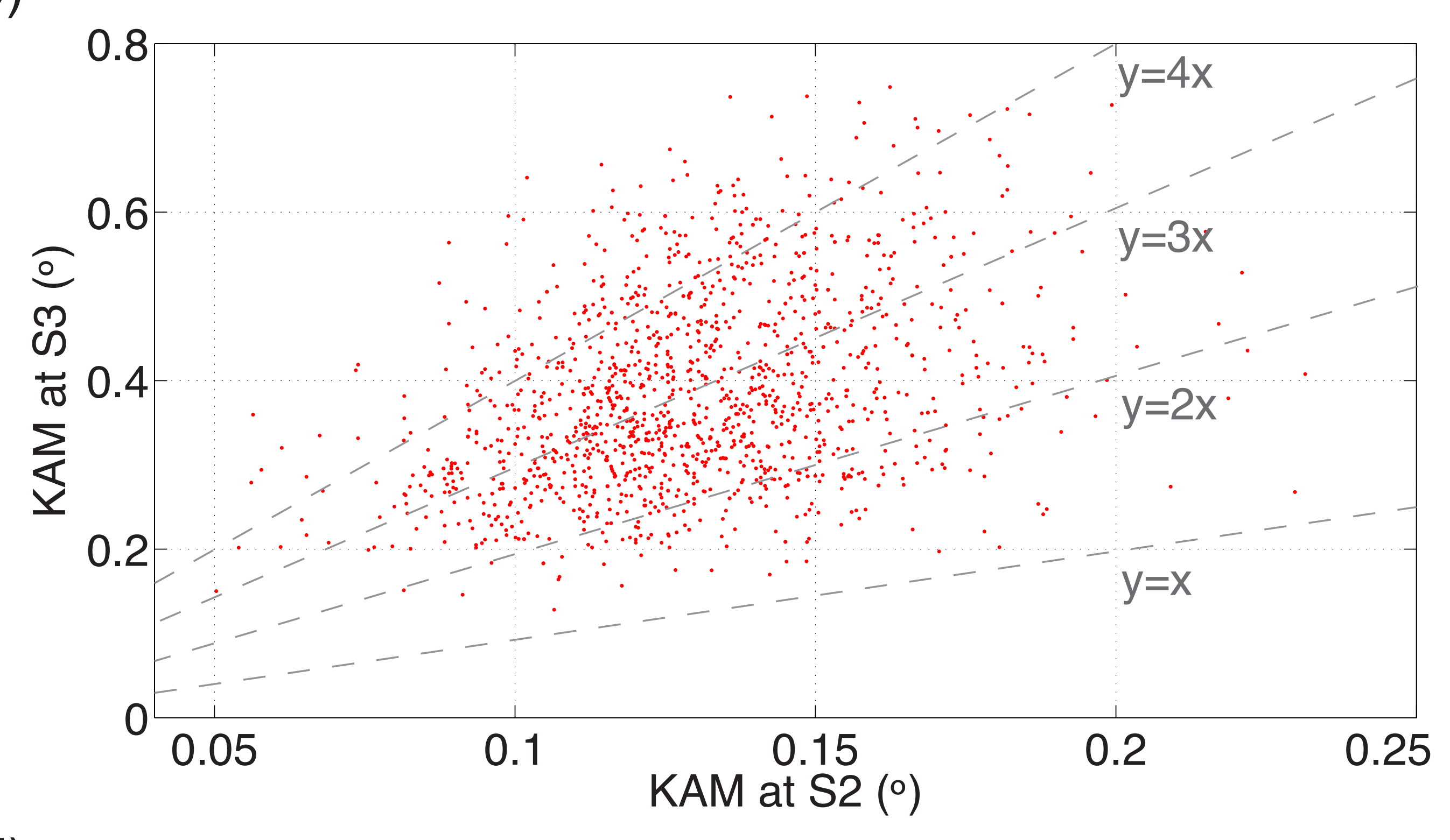

(d)

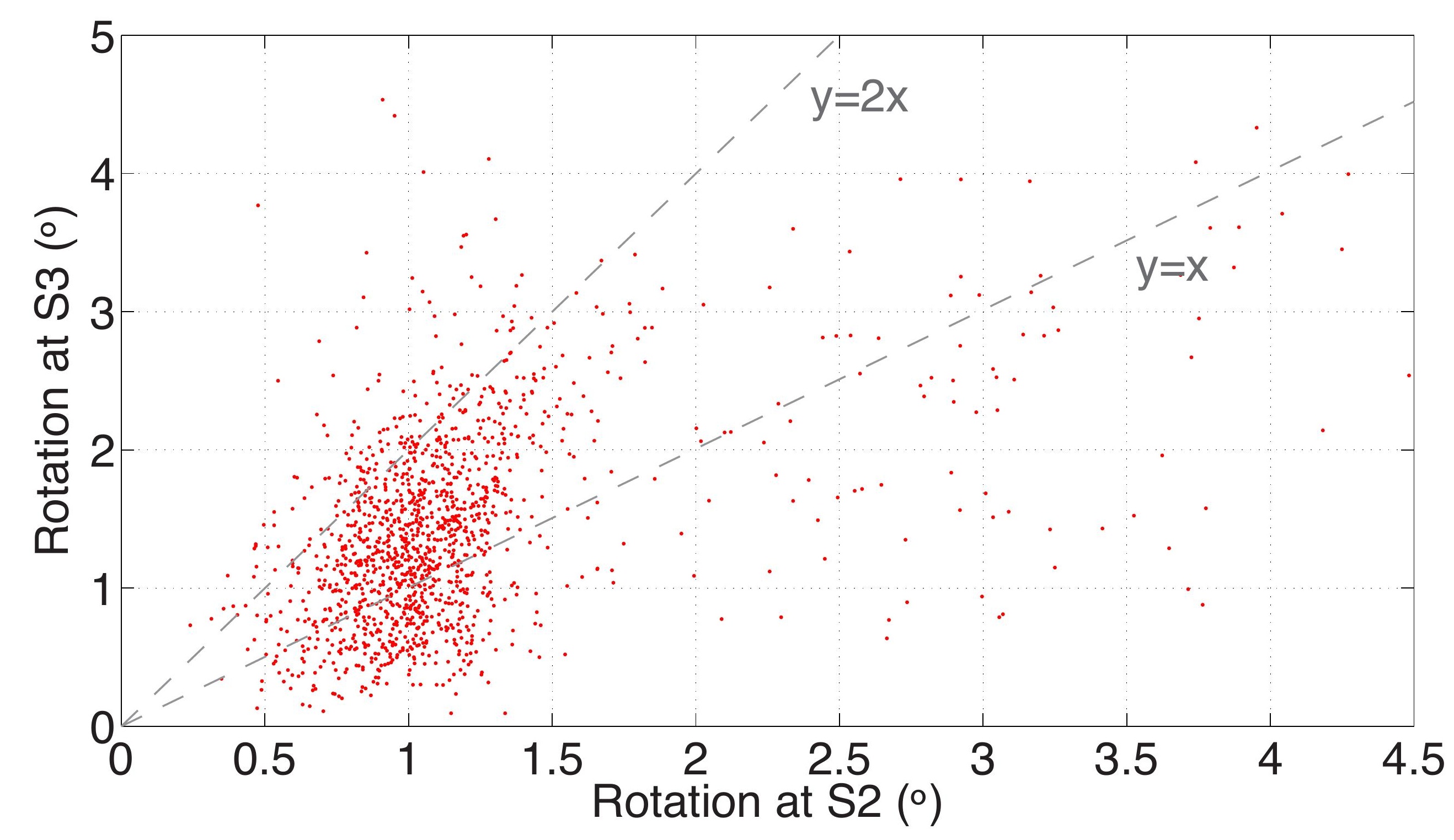


(a)

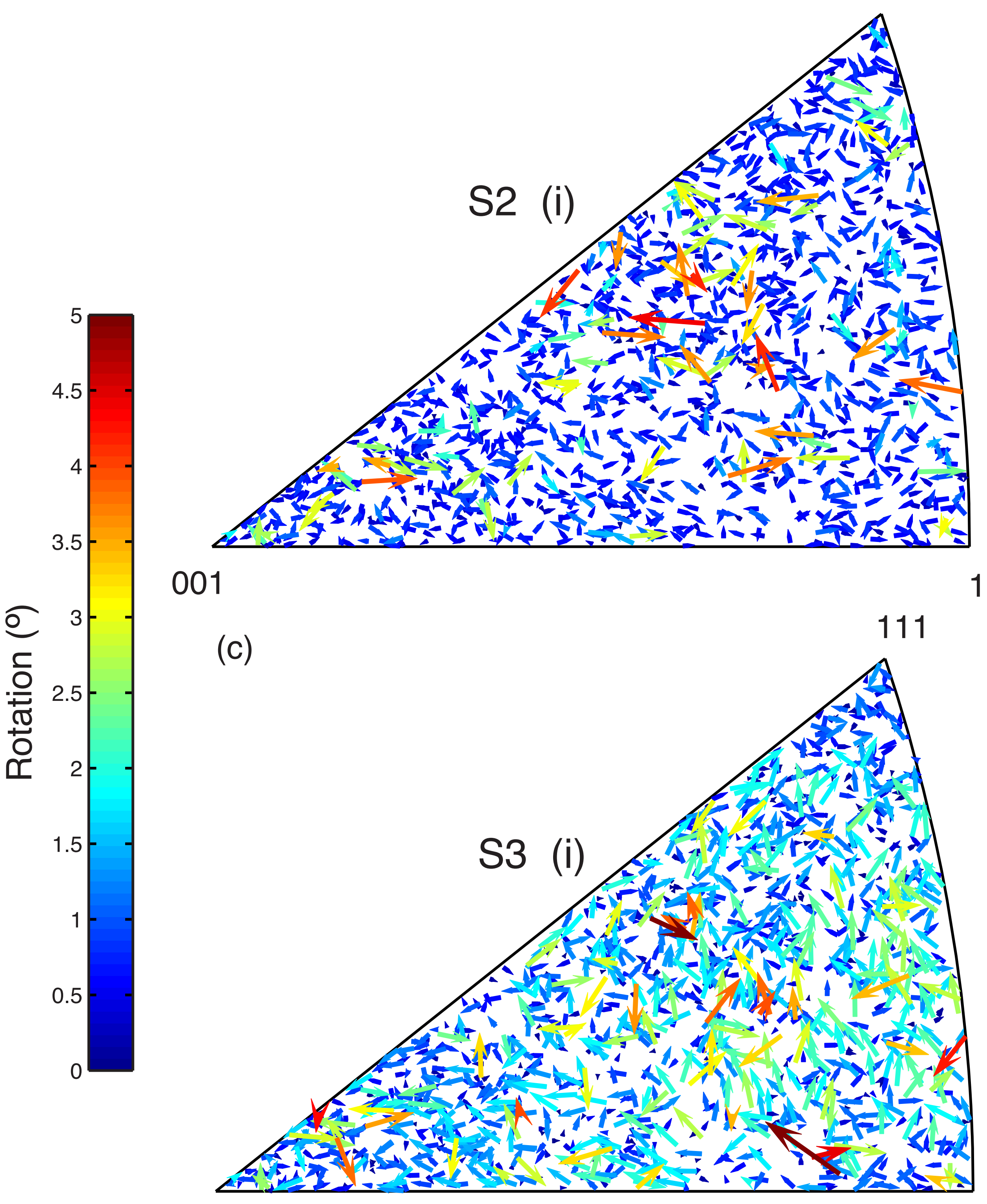

(b)

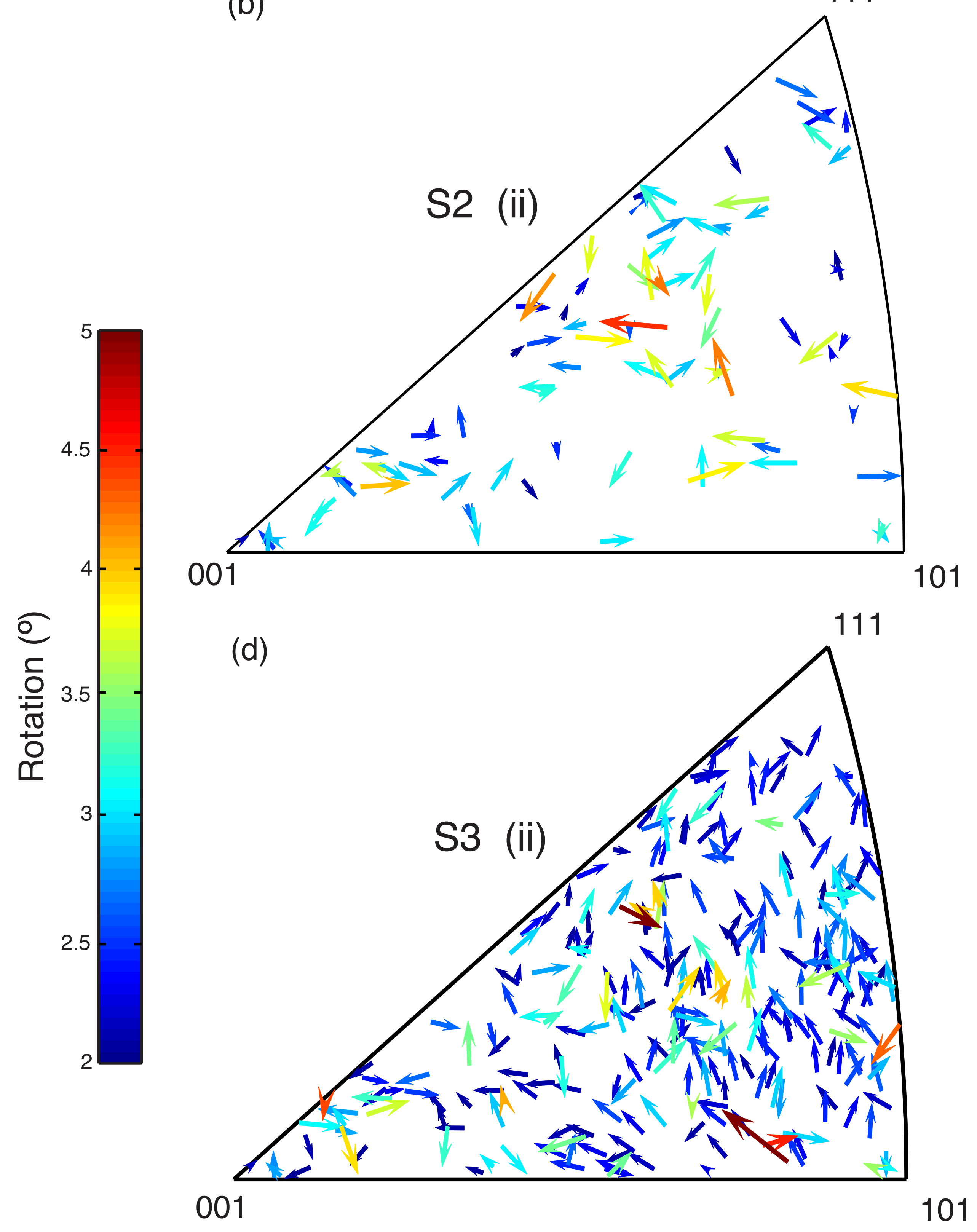




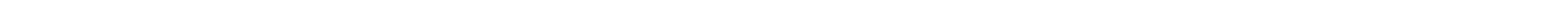


Table

\begin{tabular}{ccccc}
\hline State & $\begin{array}{c}\text { True strain } \\
(\%)\end{array}$ & Layers & $\begin{array}{c}\text { Volume } \\
\left(\mathrm{mm}^{3}\right)\end{array}$ & $\begin{array}{c}\text { Time } \\
(\mathrm{hr})\end{array}$ \\
\hline S0 & 0 & 100 & 0.31 & 10 \\
S1 & 3 & 110 & 0.35 & 11 \\
S2 & 6 & 120 & 0.38 & 12 \\
S3 & 12 & 140 & 0.44 & 14 \\
S4 & 21 & 80 & 0.25 & 11.2 \\
\hline
\end{tabular}




\begin{tabular}{|c|ccccccccc|}
\hline Grain \# & $V / V_{\max }$ & $\Delta V_{S 0 \rightarrow S 2}(\%)$ & $\Delta V_{S 2 \rightarrow S 3}(\%)$ & $\theta_{S 0 \rightarrow S 2}\left({ }^{\circ}\right)$ & $\theta_{S 0 \rightarrow S 3}\left({ }^{\circ}\right)$ & $\langle I G M\rangle_{S 2}\left({ }^{\circ}\right)$ & $\langle I G M\rangle_{S 3}\left({ }^{\circ}\right)$ & $\langle K A M\rangle_{S 2}\left({ }^{\circ}\right)$ & $\langle K A M\rangle_{S 3}\left({ }^{\circ}\right)$ \\
\hline 1 & 1.0 & 8.2 & 1.7 & 0.8 & 0.5 & 0.5 & 1.0 & 0.1 & 0.7 \\
2 & 0.44 & -13.9 & 8.1 & 1.4 & 2.7 & 0.1 & 0.8 & 0.1 & 0.7 \\
3 & 0.36 & 20.2 & -9.3 & 1.1 & 1.2 & 0.1 & 1.0 & 0.1 & 0.9 \\
4 & 0.27 & 1.1 & 14.4 & 1.0 & 1.7 & 0.1 & 0.8 & 0.1 & 0.7 \\
5 & 0.09 & -9.8 & 19.5 & 0.8 & 0.6 & 0.2 & 0.7 & 0.1 & 0.6 \\
6 & 0.08 & -30.5 & -11.7 & 0.9 & 0.7 & 0.2 & 0.7 & 0.1 & 0.4 \\
\hline
\end{tabular}

